Elaine Rocha da Luz

\title{
Predição de propriedades de gasolinas usando espectroscopia FTIR e regressão por mínimos quadrados parciais
}

\section{DissertaÇão de Mestrado}

DePARTAMENTO DE QUÍMICA

Programa de Pós-Graduação em Química 


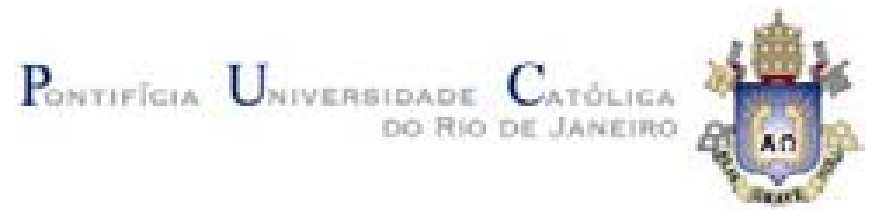

\title{
Elaine Rocha da Luz
}

Predição de propriedades de gasolinas usando espectroscopia FTIR e regressão por mínimos quadrados parciais

\begin{abstract}
Dissertação de Mestrado
Dissertação apresentada como requisito parcial para obtenção do título de Mestre pelo Programa de PósGraduação em Química da PUC-Rio.
\end{abstract}

Orientadores: Maria Isabel Pais da Silva Claudio Alberto Téllez Sotto

Rio de Janeiro, 28 de fevereiro de 2003 
Elaine Rocha da Luz

\section{Predição de propriedades de gasolinas usando espectroscopia FTIR e regressão por mínimos quadrados \\ parciais}

Prof. Maria Isabel Pais da Silva

Orientadora

Dep. de Química - PUC-Rio

Prof. Claudio Alberto Téllez Sotto

Co-orientador Instituto de Química - UFF

Ana Maria Celestino Hovell Centro de Pesquisas e Desenvolvimento - Souza Cruz S.A.

Luiz Augusto Horta Nogueira Agencia Nacional do Petróleo

Prof. Ney Augusto Dumont Coordenador Setorial de Pós-Graduação e Pesquisa do Centro Técnico Científico - PUC-Rio

Rio de Janeiro, 28 de fevereiro de 2003 
Todos os direitos reservados. É proibida a reprodução total ou parcial do trabalho sem autorização da universidade, da autora e dos orientadores.

\section{Elaine Rocha da Luz}

Graduou-se em Química na Universidade Federal do Rio de Janeiro em 1997 e em Licenciatura em Química na mesma universidade em 2000.

Ficha Catalográfica

Luz, Elaine Rocha da

Predição de propriedades de gasolinas usando espectroscopia FTIR e regressão por mínimos quadrados parciais / Elaine Rocha da Luz; orientadores: Maria Isabel Pais da Silva, Claudio Alberto Téllez Sotto. - Rio de Janeiro : PUC, Departamento de Química, 2003.

[13], 109 f. : il. ; $30 \mathrm{~cm}$

Dissertação (mestrado) - Pontifícia Universidade Católica do Rio de Janeiro, Departamento de Química.

Inclui referências bibliográficas.

1. Química - Teses. 2. Gasolina. 3. FTIR. 4. PLS. I. Silva, Maria Isabel da. II. Soto, Claudio Alberto Téllez. III. Pontifícia Universidade Católica do Rio de Janeiro. Departamento de Química. III. Título.

CDD: 540 


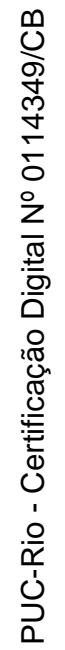

À minha mãe, por sonhar comigo. 


\section{Agradecimentos}

À CAPES pelo apoio financeiro.

Aos meus orientadores, Maria Isabel e Claudio, pela compreensão e paciência.

À Ana Hovell, por sua contribuição em minha formação profissional e pessoal.

À equipe do Laboratório de Combustíveis da PUC-Rio, principalmente Cecília, Ana Paula, Glorinha e Luiz Fernando, pelo fornecimento das amostras de gasolinas e apoio técnico.

Ao "Jorjão", técnico do Laboratório de Infravermelho, por sua ajuda na obtenção dos espectros.

Aos funcionários Carla, Fátima e Henrique, por toda a colaboração recebida.

Aos meus familiares e amigos, pelo carinho e apoio.

Ao meu marido, Fernando, por todo o incentivo. 


\section{Resumo}

Luz, Elaine. Predição de propriedades de gasolinas usando espectroscopia FTIR acoplada à regressão por mínimos quadrados parciais. Departamento de Química - PUC-Rio, 2003. 103p. Dissertação de Mestrado - Departamento de Química, Pontifícia Universidade Católica do Rio de Janeiro.

Diversas propriedades físico-químicas de gasolinas (teores de álcool, benzeno, aromáticos, saturados e olefinas, densidade, MON, RON e temperaturas de destilação) foram estimadas simultaneamente por espectroscopia na região do infravermelho médio (FTIR) acoplada à regressão por mínimos quadrados parciais (PLS). Os métodos de referência utilizados incluíram métodos ASTM, ABNT e o equipamento IROX, baseado em espectroscopia na região do infravermelho próximo. Embora o erro médio da predição (RMSEP) tenha sido o principal parâmetro considerado para selecionar o melhor modelo de predição para cada propriedade, a repetibilidade e a reprodutibilidade também foram avaliados. As propriedades que tiveram como método de referência o equipamento IROX (todos os teores, MON e RON) apresentaram, em geral, resultados mais pobres, sendo o pior resultado 0 encontrado para o teor de olefinas $(21,3 \pm 2,4)$ e o melhor para MON $(82,5 \pm$ $0,5)$. No caso das propriedades que tiveram como referência métodos ASTM ou ABNT (teor de álcool, densidade e temperaturas de destilação) o pior resultado encontrado foi para o teor de álcool $(23,9 \pm 0,7)$ e o melhor para a densidade $(0,7556 \pm 0,0025)$. No entanto, mesmo quando os valores de RMSEP encontrados através da regressão PLS foram considerados pequenos, os coeficientes de correlação $\left(R^{2}\right)$ entre os valores preditos e os de referência, para um conjunto de amostras independentes da calibração, foram menores que 0,70, para todas as propriedades. Isso se deve, principalmente, a pouca variação nos valores de algumas propriedades, como MON, por exemplo, que em todas as gasolinas analisadas apresentou valores entre 81,4 e 83,8. Ainda assim, o método FTIR-PLS mostrou-se bastante promissor como uma alternativa para a análise de gasolinas, podendo ser melhorado com a utilização de maior número de amostras de calibração e/ou com a utilização de um conjunto de amostras mais representativo, além da utilização de métodos de referência padrão.

\section{Palavras-chave}

gasolina; FTIR; PLS. 


\section{Abstract}

\section{Prediction of properties of gasoline using FTIR spectroscopy and PLS}

Several gasoline properties (alcohol, benzene, aromatics, saturated and olefin contents, density, MON, RON and distillation temperatures) have been predicted simultaneously by spectroscopy in the region of the mid infrared (FTIR) and partial least squares regression (PLS). The methods used as reference have included ASTM and ABNT methods and the IROX equipment, based on spectroscopy in the region of the near infrared. Although the standard error of the prediction (RMSEP) has been the main considered parameter to select the best model of prediction for each property, the repeatability and the reproducibility have also been evaluated. In general, the properties determined by IROX as the reference method (all the constituents, MON and RON) have presented poor results, the worst one found being the olefins content $(21,3 \pm 2,4)$ and the best one MON $(82,5 \pm 0,5)$. In the case of the properties that had ASTM or ABNT methods as reference (alcohol content, density and distillation temperatures) the worst results were found for the alcohol content $(23,9 \pm 0,7)$ and the best one for the density $(0,7556 \pm 0,0025)$. However, even values of RMSEP found by PLS regression had been considered small, the correlation coefficients $\left(R^{2}\right)$ between the predicted values and reference values, for a set of samples independent of the calibration, have been shown to be below 0,70 , for all the properties. This fact can be explained by the small variation in the values of some properties, as MON, that in all gasoline samples presented values between 81,4 and 83,8 . FTIR-PLS method revealed promising as an alternative for gasoline analysis. This method could be improved with the use of a greater set of calibration samples and/or with the use of a more representative sample set, beyond the use of standard reference methods.

\section{Keywords}

gasoline, FTIR, PLS. 


\section{Sumário}

1 Introdução 1

2 Revisão Bibliográfica 3

3 Espectroscopia no Infravermelho $\quad 9$

3.1. Princípios Básicos 9

3.2. Espectroscopia no Infravermelho por Transformada de Fourier (FTIR) 12

3.3. Espectroscopia no infravermelho por Refletância Total Atenuada (ATR) 16

4 A Gasolina 18

4.1. Tipos de Gasolinas $\quad 18$

4.2. Conceitos Básicos $\quad 19$

4.3. Especificações e Metodologias 22

4.3.1. MON e RON 25

4.3.2. Teor de Álcool 27

4.3.3. Destilação 28

$\begin{array}{ll}\text { 4.3.4. Densidade } & 28\end{array}$

5 Análise Multivariada dos Dados 30

5.1. Quimiometria 30

5.2. Organização dos Dados 32

5.3. Princípios Básicos de uma Técnica Multivariada 34

5.4. Análise dos Componentes Principais (PCA) 35

5.4.1. Posto de uma Matriz $\quad 35$

5.4.2. Autovetores e Autovalores 35

5.4.3. O Espaço de Fatores 36

5.4.4. Descrição Matemática da PCA 37

5.5. A Regressão por Mínimos Quadrados Parciais 38

5.6. Pré-tratamentos Opcionais dos Dados 42

6 Procedimento Experimental $\quad 45$

6.1. Amostras 45 
6.1.1. Misturas de Solventes $\quad 45$

6.1.2. Gasolinas $\quad 53$

6.2. Análise no Infravermelho 60

6.3. Calibração PLS 60

7 Resultados e Discussão 12

7.1. Detecção de Amostras Aberrantes 62

7.2. Seleção de Variáveis $\quad 67$

7.3. Os Modelos de Regressão PLS 70

$\begin{array}{ll}\text { 7.3.1. Misturas de Solventes } & 75\end{array}$

7.3.2. Gasolinas 83

7.3.3. Precisão do Método FTIR-PLS 101

7.3.4. Gasolinas versus Misturas 102

8 Conclusões 104

9 Referências Bibliográficas 106 


\section{Lista de figuras}

Figura 1- Tipos de vibrações.

Figura 2- Diagrama de blocos mostrando os principais componentes de um espectrômetro FTIR.

Figura 3- O interferograma de uma fonte de freqüência única ( $a$ e b).

Interferograma de uma fonte de duas freqüências (c) (2).

Figura 4- Interferograma de uma fonte de infravermelho típica (2).

Figura 5- Efeito da apodização do interferograma sobre o espectro. (a) sem

apodização; (b) apodização boxcar; (c) apodização triangular (2).

Figura 6- Representação da matriz de dados $X$ decomposta em produtos de matrizes de posto igual a um (30).

Figura 7- Um componente principal no caso de duas variáveis: (A) loadings são os cosenos dos ângulos do vetor direção; (B) scores são as projeções das amostras 1 a 6 na direção do componente principal (30).

Figura 8- Pré-processamento dos dados.

Figura 9- Planejamento experimental das misturas de solventes, ilustrando a distribuição dos teores de ciclo-hexeno e n-heptano.

Figura 10- Planejamento experimental das misturas de solventes, ilustrando a distribuição dos teores de iso-octano e etanol.

Figura 11- Planejamento experimental das misturas de solventes, ilustrando a distribuição dos teores de benzeno e tolueno.

Figura 12- Planejamento experimental das misturas de solventes, ilustrando a distribuição dos teores de meta, para e orto-xileno.

Figura 13- Espectros no infravermelho de 67 misturas de solventes, na região de 4000 a $650 \mathrm{~cm}^{-1}$, para verificação da presença de amostras anômalas.

Figura 14- Espectros no infravermelho de 126 gasolinas, na região de 4000 a $650 \mathrm{~cm}^{-1}$, para verificação da presença de amostras anômalas.

Figura 15- Gráfico dos "scores" nos três primeiros componentes principais dos espectros das misturas apresentados na figura 13.

Figura 16- Gráfico de influência dos espectros das misturas apresentados na figura 13.

Figura 17- Gráfico do "scores" nos três primeiros componentes dos espectros das gasolinas apresentados na figura 14.

Figura 18- Gráfico de influência dos espectros das gasolinas apresentados na 
figura 14.

Figura 19- Espectros no infravermelho das 66 misturas de solventes, na região de 4000 a $650 \mathrm{~cm}^{-1}$, utilizados na calibração, validação e teste do modelo PLS. 67 Figura 20- Coeficientes de regressão do modelo PLS construído com os espectros inteiros (650-1650 e $\left.2600-3100 \mathrm{~cm}^{-1} 1\right)$ das misturas de solventes para a predição de benzeno.

Figura 21- Cargas das variáveis $X$ nos componentes principais 1 e 2 do modelo PLS construído com os espectros inteiros (650-1650 e 2600-3100 cm¹) das misturas de solventes para a predição do teor de benzeno.

Figura 22- Gráfico RMSEP versus o número de componentes principais, do modelo

PLS construído para a determinação do teor de aromáticos nas misturas.

Figura 23- Espectro do etanol com as regiões $650-1500$ e $2700-3050 \mathrm{~cm}^{-1}$ destacadas.

Figura 24- Teor de aromáticos (\%m) nas misturas de solventes. 78

Figura 25- Teor de aromáticos $(\% \mathrm{~m})$ nas misturas de solventes. $\quad 78$

Figura 26- Teor de benzeno (\%m) nas misturas de solventes. 79

Figura 27- Teor de benzeno (\%m) nas misturas de solventes. 79

Figura 28- Teor de saturados (\%m) nas misturas de solventes. 80

Figura 29- Teor de saturados $(\% \mathrm{~m})$ nas misturas de solventes. 80

Figura 30- Teor de ciclo-hexeno (\%m) nas misturas de solventes. 81

Figura 31- Teor de ciclo-hexeno (\%m) nas misturas de solventes. 81

Figura 32- Teor de etanol (\%m) nas misturas de solventes. 82

Figura 33- Teor de etanol (\%m) nas misturas de solventes. 82

$\begin{array}{ll}\text { Figura 34- Teor de aromáticos (\%v) nas gasolinas. } & 87\end{array}$

Figura 35- Teor de aromáticos (\%v) nas gasolinas. $\quad 87$

Figura 36- Teor de benzeno (\%v) nas gasolinas. 88

Figura 37- Teor de benzeno (\%v) nas gasolinas. 88

Figura 38- Teor de álcool (\%v) nas gasolinas. $\quad 89$

Figura 39- Teor de álcool (\%v) nas gasolinas. $\quad 89$

Figura 40- Teor de olefinas (\%v) nas gasolinas. $\quad 90$

Figura 41- Teor de olefinas (\%v) nas gasolinas. $\quad 90$

Figura 42- Teor de saturados (\%v) nas gasolinas. $\quad 91$

Figura 43- Teor de saturados (\%v) nas gasolinas. $\quad 91$

Figura 44- Valores de MON nas gasolinas. $\quad 92$

Figura 45- Valores de MON nas gasolinas. $\quad 92$ 
Figura 46- Valores de RON nas gasolinas. 93

Figura 47- Valores de RON nas gasolinas. 93

Figura 48- Teor de álcool (\%v) nas gasolinas. $\quad 94$

Figura 49- Teor de álcool (\%v) nas gasolinas. $\quad 94$

Figura 50- Valores de densidade das gasolinas. 95

Figura 51- Valores de densidade nas gasolinas. 95

Figura 52- Valores de ponto de ebulição inicial nas gasolinas. 96

Figura 53- Valores de PE 10\% nas gasolinas. $\quad 97$

Figura 54- Valores de PE 10\% nas gasolinas. 97

Figura 55- Valores de PE 50\% nas gasolinas. 98

Figura 56- Valores de PE 50\% nas gasolinas. 98

Figura 57- Valores de PE 90\% nas gasolinas. 99

Figura 58- Valores de PE 90\% nas gasolinas. 99

Figura 59- Valores de PEF nas gasolinas. 100

Figura 60- Valores de PEF nas gasolinas. 100

Figura 61- Análise dos componentes principais (PCA) de todas as amostras de gasolinas e misturas de solventes. 


\section{Lista de tabelas}

Tabela 1-Correlação entre composição química e octanagem (8). 21

Tabela 2- Especificações para gasolinas (9). 24

Tabela 3- Normas aplicadas às gasolinas automotivas brasileiras comercializadas em todo território nacional: Normas Brasileiras (NBR) e Métodos

Brasileiros (MB) da Associação Brasileira de Normas Técnicas (ABNT) ou normas da American Society for Testing and Materials (ASTM) (9). 25

Tabela 4- Planejamento experimental das misturas de solventes (porcentagem em volume).

Tabela 5- Composição das misturas de solventes (\%m) -

amostras de calibração. $\quad 51$

Tabela 6- Composição das misturas de solventes (\%m) - amostras teste. 52

Tabela 7- Propriedades das gasolinas determinadas no Laboratório de

Combustíveis da PUC-Rio através de métodos-padrão de referência.

Tabela 8- Propriedades das gasolinas determinadas no Laboratório de combustíveis da PUC-Rio através do equipamento IROX.

Tabela 9- Modelos PLS selecionados para predição das propriedades das misturas de solventes.

Tabela 10- Modelos PLS selecionados para predição das propriedades das gasolinas.

Tabela 11- Erros relativos do conjunto teste das misturas de solventes. $\quad 76$

Tabela 12- Erros relativos do conjunto teste das gasolinas para os modelos cujo método de referência foi o IROX.

Tabela 13- Erros relativos do conjunto teste das gasolinas para os modelos cujos métodos de referência foram ASTM ou ABNT.

Tabela 14- Repetibilidade e reprodutibilidade do método FTIR-PLS para predição de propriedades de gasolinas comerciais. 